\title{
Il testo (non) è mobile
}

\section{Mario Ricciardi, Politecnico di Torino}

Abstract: The framework for this contribution is the transformation of the textual community, namely, modern society, into a digital community, in other words, a network society. This paper analyzes two theses. The first holds that the text, due to its genetic and historic nature, is always IMMUTABLE, STABLE, and never mobile. For this reason, the text represents the foundational element of a specific society, modern society. The second thesis is based on the assertion that within information and hypertext technologies two diverse views confront and contrast each other. I locate these tendencies in the following key words: "dream" (Nelson) and "intellect" (Engelbart). Hypertext technology and culture represent the most transformative location and agency, they are the actors of the paradigm shift in which we are implicated. The hypertext revolution is based on the criticism of and attack against the material supports of writing and reading, as well as typographic technologies. Hypertext fights and wins this battle. However, hypertext technology is not able to reach the deep structure of alphabetic culture that is made not only of material support but also mental and bodily experience, consolidated and evolving, and of social relationships that are implied in the materiality of the support.

La cornice di riferimento di questo contributo è la trasformazione della società testuale, cioè della società moderna, in società digitale, ovvero nella società dei network. Nell'intervento vengono analizzate due tesi: la prima afferma che il testo, per sua natura genetica e storica, è sempre IMMUTABILE, STABILE e non è mai mobile e per questo è fondativo di una società specifica, quella che noi chiamiamo la società moderna. La seconda tesi si basa sull'assunto che all'interno della cultura informatica e tecnologica che genera gli ipertesti si confrontano e si scontrano due idee diverse. Identifico queste due tendenze nelle parole chiave: dream (Nelson) e intellect (Engelbart). La tecnologia e la cultura ipertestuale sono il luogo e l'agente più fortemente trasformativi, gli attori del cambio di paradigma che ci coinvolge. La rivoluzione ipertestuale ha le sue basi nell'attacco e nella critica dei supporti materiali della scrittura e della lettura e sulle tecnologie tipografiche. L'ipertesto combatte e vince questa battaglia. La tecnologia ipertestuale non riesce però a raggiungere la struttura profonda della cultura alfabetica che è composta non solo da 
supporti ma da esperienze sia della mente sia del corpo, da relazioni sociali consolidate e mutanti che sono sottese e operanti con la materialità del supporto.

\section{Premessa}

L'occasione dell'incontro a Roma il 10 gennaio 2012, intitolato “Il testo è mobile”, è stato per me particolarmente utile e interessante. Il tema proposto mi ha ricondotto a ricerche e studi di un periodo, fine degli anni Ottanta inizio degli anni Novanta del secolo passato, dedicati alla nascita delle tecnologie ipertestuali e alla loro evoluzione. Sono ritornato a riflettere su un tema che mi è particolarmente caro e cioè la trasformazione della società testuale, cioè della società moderna, in società digitale, nella società dei network. E metto volutamente l'accento sul termine società, perché mi sembra molto più comprensivo e convincente di altri, come rivoluzione digitale. Termini che troppo insistono solo sulla dimensione tecnologica e che presentano in modo troppo compatto e uniforme l'informatica, dimenticando le tensioni e le diverse posizioni che invece si contrapponevano in quel periodo. L'ipertesto è il punto chiave, ed è anche una scelta terminologica assolutamente decisiva: rappresenta un momento alto di sintesi tra il mondo umanistico e quello tecnologico. Ma anche su questo incontro-scontro ci sono -come vedremo- opinioni diverse.

Mi propongo quindi di sostenere due tesi:

La prima ha origine dall'assunto provocatorio, opposto al titolo del convegno, e cioè che il testo per sua natura genetica e storica è sempre IMMUTABILE, STABILE e non è mai mobile. Quindi tutto ciò che è mutevole e indefinito non è testo. Per semplificare userò la parola TEXTUS per indicare la struttura profonda, eterna, se misurata col tempo storico delle civiltà moderne che si sono succedute nel Mediterraneo e in Europa. La parola testo invece ha assunto un valore onnicomprensivo e buono a tutti gli usi, ma appunto si tratta di usi, di movimenti di superficie.

La seconda tesi si basa sull'assunto che all'interno della cultura informatica e tecnologica che genera gli ipertesti si confrontano e si scontrano due idee diverse che pure nei primi anni Novanta del secolo passato si richiamano a una autorevole e comune matrice, rappresentata da Vannevar Bush. Identifico queste due tendenze nelle parole chiave: dream (Ted Nelson) e intellect (Douglas Engelbart).

Io sono sostenitore del valore positivo del conflitto dentro la società; per me è il conflitto che genera sia i processi rivoluzionari o i cambi di paradigma sia l'emergere visibile di posizioni e di interessi diversi e spesso divergenti. Al contrario oggi è dominante una forma di pensiero egemone che è stata etichettata, forse in modo semplicistico, come pensiero unico. Personalmente preferisco parlare di cultura conciliante, cioè della ricerca di forme assolutorie, che negano appunto il conflitto o meglio ancora lo dissolvono nella pacificazione delle parti in causa e infine lo neutralizzano. In realtà è sufficiente vedere come sono andate e stanno andando le vicende del mondo per dedurne che una sola parte è costantemente vincente (almeno negli ultimi venti-trenta anni). 
Nell'incontro mi ero proposto di affrontare due temi: uno è appunto quello che va sotto il titolo: il testo NON è mobile, l'altro invece era indicato così: "inventare il passato”. Di questo secondo tema non mi è possibile, per ragioni di spazio, parlare. Mi limito a dire che intendevo sviluppare questi argomenti: qual è il canone prevalente nella letteratura italiana? È quello identificato nella storia letteraria e in particolare nella figura di Francesco De Sanctis? Scegliendo alcuni esempi sia della storia letteraria sia dei saggi intendevo dimostrare che non è così. Il vero codificatore del canone è Pietro Bembo fortemente sorretto dalla tecnologia gutemberghiana che fonda davvero le regole e i modelli. De Sanctis in realtà sfugge da questo metodo: gli esempi che avevo scelto sono alcuni dei personaggi chiave del suo modo di fare critica e storia: Machiavelli e Leopardi. Ma di questi argomenti -speroavremo occasione di parlarne un'altra volta.

\section{Il testo (non) è mobile perché ciò che è mobile non è testo}

Questo sillogismo che come tutti i sillogismi - per sua natura - evita di confrontarsi con le prove e le verifiche critiche è utile per fare emergere meccanismi e contraddizioni che nell'uso corrente della parola "testo" sembrano scomparsi; propongo perciò di distinguere tra textus e testo.

L'opposizione tra textus e testo è utile per mettere in evidenza processi che si muovono parallelamente: il primo appare vincente a partire dalla fine degli anni Ottanta- inizio anni Novanta, quando appaiono i primi software ipertestuali creati per i due ambienti dominanti MS-DOS e Mac. In questo caso, come vedremo, gli innovatori (cioè non soltanto i tecnologi ma anche gli umanisti che cooperano per realizzare e diffondere gli ipertesti) hanno estrema fiducia nell'azione "rivoluzionaria" di questa tecnologia per spezzare il potere del testo, dissolvendolo insieme al modello dominante: quello logico-sequenziale. L'attenzione si concentra perciò sul ruolo dei supporti materiali e sulle tecnologie tipografiche. L’ipertesto combatte e vince questa battaglia.

La distinzione tra textus e testo ci consente di osservare un curioso processo: il textus in realtà non viene intaccato. La tecnologia ipertestuale non riesce a raggiungere questa struttura profonda che è composta non solo da supporti, ma da esperienze sia della mente sia del corpo, da relazioni sociali consolidate e mutanti che sono sottese e operanti con la materialità del supporto. Contemporaneamente la parola testo ha una diffusione tale da essere considerata sinonimo di documento e viceversa; in questo modo si registra nei fatti una fusione tra il lessico informatico e quello umanistico. Una fusione che privilegia l'uso.

Il textus al contrario è indice di una presenza latente, che non emerge in questi processi, ma che fa capo alla struttura profonda delle relazioni tra gli esseri umani, alla comunicazione e alle relazioni sociali. Questo mondo non affiora immediatamente in questi processi, ma si farà strada come sapere laterale, come cultura condivisa non appena gli ipertesti usciranno dalla dimensione locale e saranno messi in rete, sconvolgendo anche il meccanismo tipico delle origini di Internet (gli accessi, i percorsi, la navigazione seguivano il modello logico-sequenziale) per poi trasformarsi in ipermedia e dare quindi spazio strategico all'immagine. La dimensione sociale che si afferma riduce drasticamente il ruolo 
del documento e del testo sia per la rilevanza che assumono le connessioni sia per il costituirsi delle comunità virtuali in cui il valore dell'esperienza condivisa riduce il valore dell'oggetto da cui questa esperienza trae origine.

Dobbiamo dunque distinguere tra l'uso corrente della parola "testo" e il significato originario di textus. È particolarmente utile a questo proposito porre l'accento sulla genesi del textus, sul processo che produce la rivoluzione alfabetica e quindi la mentalità alfabetica (secondo Havelock).

In sintesi, per il nostro discorso, possiamo individuare le posizioni più significative:

- una visione conciliante: lo scioglimento dell'oralità nell'alfabetizzazione. Processo naturale ed evolutivo;

- una visione logocentrista: superamento dell'oralità nella scrittura alfabetica, i primitivi superati dai civilizzati (cioè gli alfabetizzati). La scrittura alfabetica è il superamento dell'oralità, cioè dell'assenza di supporto materiale, memoria fissa e stabile, di riproducibilità e di sequenzialità vincolante (significato);

- una visione conflittuale: la fine dell'oralità è una caduta, una perdita irreparabile. La scrittura e poi soprattutto la stampa sono un processo di alienazione - proprio nel senso di Marx dei manoscritti economico-filosofici -, uno spossessamento dell'umano e delle sue relazioni sociali assoggettato contemporaneamente a processi di astrattizzazione (logica testuale) e di cosificazione (la produzione seriale attraverso la tipografia).

Il punto di resistenza alla mobilità e alla leggerezza e alla fluidità fino a rendere queste qualità meri accidenti, attributi irrilevanti, è il textus e il suo rapporto organico col supporto che lo fa vivere pienamente e lo rende vivente non solo per sé come un materiale qualsiasi, ma oggetto dedicato, straordinariamente specializzato nella comunicazione e nell'attivazione sia dei processi sociali sia delle attività mentali.

Come nasce il textus? Per opposizione alla parola parlata e come evoluzione del discorso in presenza verso forme più complesse e differite nel tempo e rivolte a interlocutori estranei a chi pronunciava quelle parole. Appare evidente la sua funzione di medium, di mediatore sociale e di generatore di tempo differito, necessario alla riflessione.

Per Ong la scrittura è un atto di colonizzazione tecnologica degli spazi aperti della parola, una perdita della naturalità innata del parlare diretto alla comunità di chi ascolta. La tecnologizzazione della parola deve necessariamente approdare a forme di standardizzazione, di serialità che al tempo stesso producono allontanamento dalla spontaneità vitale dei parlanti (e dei viventi) e insieme forme astratte e simboliche di produzione, ri-produzione e comunicazione del pensiero. La serialità alfabetica trova la sua massima espressione nella tecnologia tipografica, nell’officina di Gutenberg dove nascono contemporaneamente la produzione in serie e l'alienazione. Secondo Ong, Marx si limita al rapporto di produzione, al rapporto materiale di produzione, per definire l'alienazione dell'uomo nella società capitalistica. Ma il processo di spossessamento e di perdita della 
totalità dell'umano è già avvenuto molto tempo prima. Ed è fondamentalmente una caduta dal tempo in cui la Voce poteva essere ascoltata direttamente e senza mediatori.

Il passaggio dall'oralità alla competenza scritturale, è determinato dalla tecnologizzazione della parola, cioè da una forma di linguaggio naturale, diretto, comunitario, a una forma di linguaggio in cui la tecnica acquista un ruolo determinante. È l'opposizione tra naturale e artificiale (o innaturale come diranno alcuni dei protagonisti della nuova cultura ipertestuale). E la tecnologia è sempre e comunque artificiale, è un qualcosa di materiale che sta fuori di noi La parola perde quella funzione che le consentiva di essere l'elemento fondante della comunità; la tecnologia della scrittura e la tecnologia della stampa costruiscono società e non più comunità, dove la mediazione tra gli esseri umani e i complessi meccanismi psicologici e sociali che vengono messi in moto sono i vettori di una nuova figura dell'io: l'individuo moderno.

\section{II rapporto tecnologia-scrittura: da Platone agli ipertesti}

Ong rinvia al celebre passo del Fedro sottolineando l'origine precoce della riflessione sull'ambiguità della scrittura rispetto alla piena trasparenza della parola. La parola è spirito e la scrittura è tecnologia.

Platone pensava alla scrittura come a una tecnologia esterna, aliena, nello stesso modo in cui oggi molte persone pensano al computer. Noi invece oggi l'abbiamo ormai interiorizzata così profondamente, l'abbiamo resa una parte tanto importante di noi stessi, che ci sembra difficile pensarla come tecnologia al pari della stampa e del computer. Ciò nonostante, la scrittura (e in special modo quella alfabetica) è una tecnologia, che richiede l'uso di una serie di strumenti quali penne stilografiche, pennelli o biro, superfici predisposte come la carta, pelli di animale, tavolette di legno, e inoltre inchiostro, colori, e molte altre cose (...). La scrittura, sotto certi aspetti, è la più drastica delle tre tecnologie di cui abbiamo parlato. Essa dette inizio a quanto la stampa e i computer hanno poi portato avanti: la riduzione del suono a spazio, la separazione della parola dal presente immediato e vivo, nel quale possono esistere solo parole parlate. Al contrario del linguaggio naturale, orale, la scrittura è del tutto artificiale: non c’è modo di scrivere "naturalmente". Il discorso parlato è invece sentito come naturale dagli uomini nel senso che, in ogni cultura, chiunque non abbia danni fisici o psichici impara a parlare. Il parlare permette la vita cosciente, ma sale alla coscienza da profondità inconsce, seppure con la cooperazione - consapevole o meno - della società. (Ong 123)

Anche l'antropologo Jack Goody è consapevole del conflitto tra parola e cosa così come si manifesta sotto la forma tipica della visione del capitalismo occidentale: ovvero l'economia è il primum e la parola comunicata e quindi la scrittura alfabetica viene di conseguenza. Il capitalismo industriale accentua il primato dell'economia parallelamente all'invenzione della stampa e alla diffusione del libro. Ma Goody scopre che tra gli agenti che costituiscono la società viene prima la comunicazione e poi la produzione: affermazione 
sconvolgente e forse temeraria. Esprime un punto di vista pericolosamente estraneo rispetto al pensiero dominante della società del capitalismo occidentale:

La cultura è un insieme di atti di comunicazione, e le differenze nel modo di comunicazione sono spesso altrettanto importanti delle differenze nel modo di produzione, implicando sviluppi sia nell'accumulazione, nell'analisi e nella creazione del sapere umano sia nelle relazioni tra gli individui che ne sono interessati. (Goody, Il suono e i segni, 48)

\section{Alla fine Goody attenua alcune affermazioni drastiche e rivede le sue posizioni estremistiche:}

Le dinamiche della comunicazione e i processi di relazione tra gli individui appaiono caratterizzati da un'alternanza tra mutamenti continui (evoluzione, ma anche rottura) e presenze spesso nascoste o latenti per un lungo periodo (tradizione, memoria). La [...] finalità della mia analisi era spostare un pò quell'accento, troppo spesso e troppo marcatamente posto sui mezzi e sui rapporti di produzione, sui mezzi e sui rapporti della comunicazione. Con ciò non intendo solo riferirmi alle tecniche ma anche alla tecnologia, ivi compresa la tecnologia intellettuale che la comunicazione scritta attiva direttamente, si tratti dell'accumulazione della conoscenza nelle biblioteche o della conoscenza interna, sino agli elementi di costrizione e di libertà che gli esseri umani annettono a questi sistemi. [...] Benché le società non possano essere ridotte a sistemi di comunicazione e di scambio, è abbastanza ovvio che esse cambino in relazione ai cambiamenti di questi sistemi: cambiamenti in cui rientrano la monetizzazione e l'alfabetizzazione. (Goody, La logica della scrittura, 206)

Nella rivoluzione indotta dall'invenzione e poi dalla diffusione degli ipertesti è stato sopravvalutato il ruolo della tecnologia in atto e la dissolvenza del supporto, ma è stata sottovalutata la rivoluzione culturale che insieme si stava compiendo: l'indebolimento e poi l'abbandono del modello logico-sequenziale. Scelgo come punto di riferimento il momento in cui avviene la diffusione su larga scala e attraverso applicativi disponibili al largo pubblico, nei primi anni Novanta del secolo passato e mi riferisco ai due prodotti principali: HyperCard per Mac e Guide per ambiente MS-DOS ${ }^{1}$. In realtà il successo fu principalmente per HyperCard.

L'attenzione degli innovatori, dei fondatori delle tecnologie e culture ipertestuali si concentra solo su questo livello proprio della modernità, non vede la struttura profonda, permanente che sta sotto. Potremmo dire che, se viene colta la materialità dell'insieme che unisce testo e significato, un cambio di tecnologia sembra essere il grimaldello potente per scardinare il potere del testo, quel potere che sta tutto al centro, il potere del logos (Derrida). De-centrare e quindi togliere potere, autorità al testo deriva necessariamente da un'azione tecnologica che frantuma fisicamente il documento scritto e può ricomporlo in forme e unità imprevedibili solo attraverso l'intervento di un lettore trasformato in co-autore. 


\section{Il testo di tutti}

La semiologia (Barthes) è responsabile sia della leggerezza e della diffusione onnicomprensiva della parola testo sia della sua estensione a tutte le forme di comunicazione. Derrida ne propizia (involontariamente!?) la predisposizione e la piena disponibilità all'incontro con l'informatica in modo tale che la resistenza delle strutture testuali si addomestichi e si sciolga nella fluidità necessaria per accompagnare la nascita degli ipertesti.

Il più noto sostenitore dell'incontro "naturale" tra informatica e semiotica, tra tecnologie della comunicazione e post-strutturalismo, è George Landow che intitola un capitolo del suo libro dedicato agli ipertesti: "Derrida ipertestuale, Nelson poststrutturalista?”:

Quando i progettisti di software esaminano le pagine di Glas o di Della grammatologia, si imbattono in un Derrida digitalizzato e ipertestualizzato; quando i teorici della letteratura studiano Literary Machines incontrano un Nelson decostruzionista o poststrutturalista. Queste sbalorditive sovrapposizioni possono aver luogo perché negli ultimi decenni la teoria letteraria e gli studi informatici sugli ipertesti, due aree di ricerca apparentemente non collegate, si sono avvicinate sempre più. Le affermazioni degli studiosi di letteratura mostrano oggi una notevole convergenza con quelle degli informatici. A parer mio, negli scritti di Jacques Derrida e Theodor Nelson, di Roland Barthes e Andries van Dam ha iniziato a manifestarsi un mutamento di paradigma. ... Tutti e quattro, come molti altri studiosi di teoria letteraria e di ipertesti, sostengono che dobbiamo dimenticare i sistemi concettuali basati sull'idea di centro, margine, gerarchia e linearità, e sostituirli con quelli di multilinearità, nodi, collegamenti e reti. Quasi tutte le parti coinvolte in questo mutamento di paradigma, che segna una rivoluzione nel pensiero umano, vedono nella scrittura elettronica una risposta ai vantaggi e svantaggi del libro stampato. E questa risposta ha profonde implicazioni nell'ambito della letteratura, dei sistemi educativi e della politica. (Landow 21-22)

Sempre in Landow viene sottolineato (e fatto proprio) il passaggio, rovesciato rispetto a Ong, dall'artificiosità della tecnologia tipografica alla naturalità del mezzo elettronico (perché rappresenta in modo più trasparente il pensiero e le attività mentali secondo il suggerimento di Vannevar Bush):

Come osserva J. David Bolter nel mostrare che l'ipertestualità incarna l'idea poststrutturalista di testo aperto, "ciò che è innaturale nella stampa diventa naturale nel mezzo elettronico, e presto non ci sarà nemmeno più bisogno di parlarne, perché lo si potrà mostrare.” (Landow 22; citazione Bolter 183)

Ma rimane sempre privilegiata la teoria della naturale risoluzione delle teorie testualiste (come in $S / Z$ ) nella pratica ipertestuale. La teoria sta in Barthes, l'applicazione, invece, nelle tecnologie ipertestuali. In $S / Z$ Roland Barthes (Landow 120-123) descrive una testualità ideale che corrisponde precisamente a quello che in seguito è stato chiamato ipertesto: 
Testo composto da blocchi di parole (o immagini) connesse elettronicamente secondo percorsi molteplici in una testualità aperta e perpetuamente incompiuta descritta dai termini collegamento, nodo, rete, tela e percorso (Landow 72).

Il cambio di paradigma si realizza attraverso le tecnologie ipertestuali, senza Hypercard e Guide non ci sarebbero stati ipertesti. Nell'ipertesto la tecnologia informatica permette di scomporre fisicamente il documento in tante parti ri-connettibili secondo percorsi imprevisti o non determinati totalmente dall'autore. Questa è la causa della crisi e della dissolvenza della struttura logico-sequenziale all'origine del textus, quella struttura che univa materialmente e intellettualmente (cognitivamente) il supporto materiale ai significati scambiati tra autore e lettore. Essi si costituivano come attori fondamentali della civiltà moderna fino alla svolta del nuovo paradigma, quello della tecnologia gutemberghiana che genera il rapporto indissolubile tra tecnologia meccanica e riproduttiva, e testo.

\section{Società digitale vs società del testo}

Quando parlo di società del testo intendo dire che il testo esercita una funzione organizzativa per i soggetti sociali, cioè li collega, li tiene insieme. Socialmente vale l'affermazione della vulgata strutturalista: tout se tient. Tutto socialmente deve stare insieme, altrimenti la società si dissolve, crolla, perde la sua razionalità intrinseca, il suo significato. Il collante primario della società moderna sta nell'archetipo originario del rapporto di autorità tra autore e lettore, versione culturale su cui insiste l'archetipo hegeliano di potere tra servo e padrone. Il testo non è soltanto il mezzo per rappresentare e quindi comunicare ai cittadini le dinamiche e le relazioni sociali, ma genera lo spazio sociale necessario per mediare tra gli interessi e i soggetti coinvolti: è il medium sociale.

\section{1 Ipertesti e testi}

Prendiamo come esempio l'antologia curata da Delany e Landow, Hypermedia and Literary Studies. Può essere considerato particolarmente significativo del rapporto tra tecnologie ipertestuali (che già si confrontano con i media) e la letteratura. Nel contributo iniziale dei due curatori, dal titolo: "Hypertext, Hypermedia and Literary Studies: the State of the Art”, leggiamo:

Il testo scritto è la testimonianza stabile del pensiero e per raggiungere questa stabilità il testo dovette essere basato su di un mezzo materiale: argilla, papiro o carta; tavoletta, rotolo o libro. Ma il testo è più della mera ombra o traccia di un pensiero già formato. In una cultura letterata le strutture testuali che si sono evolute nei secoli determinano il pensiero quasi con la stessa forza della struttura primaria che dà forma ad ogni espressione, la lingua. Fin tanto che il testo è stato associato ad un elemento fisico, lettori e scrittori hanno dato per scontati tre attributi cruciali: che il testo fosse lineare, delimitato e fisso. Generazioni di studiosi e autori hanno interiorizzato queste qualità come regole del pensiero ed esse hanno avuto conseguenze sociali pervasive (Delany e Landow 3; trad. mia)

Questo incipit può essere considerato il punto di partenza su cui agiscono gli ipertesti. Un punto di partenza che recepisce l'origine del textus determinata dalla sua materialità. 
Questa materialità influenza (determina) anche il modo di pensare. Fintantoché si mantiene questo rapporto si mantengono gli attributi permanenti del testo. L'ipertesto agisce per modificare questi attributi e quindi non soltanto il modo di comunicare ma anche il modo di pensare. ${ }^{2}$

Possiamo sintetizzare così il nostro percorso: il supporto materiale veicola la scrittura e crea contemporaneamente un modello artificiale talmente forte da condizionare il nostro modo di pensare. Contro l'idea di Havelock, sintetizzabile nella formula della mente alfabetica (cioè di una seconda natura che caratteriza gli alfabetizzati), occorre spezzare la gabbia di Gutenberg, liberarsi dalle catene materiali che legano testo scritto e idee. Il supporto è inanimato ma vincola ogni forma di espressione scritta. La galassia Gutenberg appare come la gabbia tipografica:

Vincolante e innaturale, essa produce il modello costrittivo basato sulla legge logico-sequenziale. È inevitabile che la tecnologia ipertestuale enfatizzi, per contrasto, i suoi risultati principali: la scompaginazione fisica del testo, la collaborazione del destinatario (ex lettore) e la produttività concentrata sui link e sulle connessioni. Il valore positivo della stabilità, della durata, che era alle origini della scrittura alfabetica, ancor prima di Gutenberg, scompare. Non si può più esclamare con Orazio: exegi monumentum aere perennius (Odi III, 30).

Tale costellazione di riferimento della "rivoluzione ipertestuale" si appoggia sulla vision di Vannevar Bush: associazionismo mentale, sapere latente e diffuso (lo spazio di relazione e laterale tra gli utenti fino allo User Generated Content), ma insieme viene affermato il primato della ratio astratta: think, mind, intellect. "Come possiamo pensare" è il riferimento fondamentale a cui fanno omaggio sia a Doug Engelbart sia Nelson. Le associazioni mentali sono naturali, la visualizzazione e la comunicazione alfabetica (ancor più la sua versione a stampa) innaturali.

Engelbart si rivolge ai programmatori come destinatari, intende occupare lo spazio simbolico di relazione tra i caratteri della scrittura, la rete di comunicazione a distanza (un concetto, non uno spazio fisico misurabile dalla lontananza). La parola chiave è augmenting, l'obbiettivo è la collaborazione tra computer e intelligenza.

Nelson invece difende la sua origine artistica e crede nella creatività dei media (teatro e cinema). Ipertesto e letteratura rappresentano non solo la mente, ma anche il corpo ed è la letteratura che favorisce concetti come dream e vision, apertura e pluralismo. Infine Nelson è contro il Web 2.0, l’informatica canonica e la cultura conciliante.

\subsection{As we may think ${ }^{3}$}

Nel 1991 si celebra il ruolo di precursore di Vannevar Bush nell'ideazione e realizzazione degli ipertesti; ma Engelbart da un lato e Nelson dall'altro hanno idee diverse, privilegiando l'uno l'augmenting e l'intellect (parola chiave anche in Bush) l'altro il sogno (dream), la visione che ha il suo fondamento in un'idea profonda della letteratura (literacy). Secondo Bush 
La mente umana [...] opera per associazioni. Una volta che essa abbia un elemento a disposizione, essa salta istantaneamente all'elemento successivo suggerito, in base a un intrico di piste registrare nelle cellule del cervello, dalla associazione dei pensieri. Naturalmente, la mente ha anche altre caratteristiche. Piste che non vengono frequentemente percorse tendono a svanire, gli elementi non sono permanenti, la memoria è transitoria. Eppure, più di ogni altra cosa in natura, la velocità di reazione, la complessità delle interrelazioni, il dettaglio delle immagini mentali, incutono meraviglia. (Bush 1945 trad. it. in Nelson Literary Machines, 1/49)

Bush esaminando il funzionamento della mente umana mette in rilievo la sua particolarità di procedere per associazioni cioè in una forma non lineare e non sequenziale; profondamente diversa dallo strumento artificiale che caratterizza tutti i prodotti culturali del genere umano nella modernità: l'alfabeto, la scrittura, infine la stampa e dunque la forma libro all'interno della quale sono contenuti tutti i documenti e tutti i messaggi prodotti. La mente procede con meccanismi non-lineari e quindi naturali; la stampa è un medium che serializza questi processi per comunicare, così come aveva fatto la scrittura alfabetica. La sintesi attuale dei processi di tecnologizzazione della mente umana e delle sue espressioni sensibili attraverso la produzione e comunicazione di documenti è la forma testuale, il testo.

Bush contrappone così i meccanismi naturali dell'umano alle forme materiali esistenti di produzione, conservazione e fruizione dei documenti scritti e stampati. Il percorso associativo è diverso dal modello logico-sequenziale proprio dell'alfabeto occidentale e del testo stampato in libro o documento. La necessità di ripensare il funzionamento "naturale" della mente umana si colloca all'interno di una previsione generale sul futuro della società: Bush scommette sulla società dell'informazione, cioè prevede una società in cui si genera un accumulo continuamente crescente di informazioni e di documenti. A fronte di questa previsione Bush vede una sostanziale inadeguatezza della mente umana: essa deve essere almeno supportata, fortemente aiutata dalle tecnologie disponibili. Il problema fondamentale non è più soltanto la memoria statica, ma una memoria dinamica in cui siano conservabili e utilizzabili non solo i documenti fissi ma anche le connessioni e i continui riferimenti, relazioni, link che gli utilizzatori di questo immenso patrimonio, distribuito in forma ineguale in tutto il mondo, generano col loro continuo uso. La mente umana non è in grado di competere su questo terreno e non è in grado di rispondere a questa sfida.

Questa è l'origine “teorica” di Memex. La teoria è fondata da un lato sull'idea dell'inadeguatezza culturale degli strumenti per affrontare la complessità del mondo a venire: la tecnologia è necessaria soprattutto per esercitare funzioni selettive e organizzative rispetto all'esplosione prevedibile della società dell'informazione. E questa è una visione straordinaria ed è l'eredità più visibile e conosciuta di Vannevar Bush. Esiste anche una dimensione che appare operante molto tempo dopo: ponendo al centro della comunicazione la scrivania personale e mettendo l'accento sui link e sul recupero dei messaggi di scambio tra gli utenti, Vannevar Bush riconosce -sia pur con molta prudenza - un sapere e uno spazio della conoscenza che si basa sull'associazione e sulla distribuzione e condivisione di 
risorse che fuoriesce dal meccanismo della trasmissione culturale e dell'informazione, proprio dell'età della stampa e dei mass media.

Bush scopre un valore rimasto latente e pressoché sconosciuto; all'esperienza che si accumula leggendo e riflettendo singolarmente sui libri, sui testi, si aggiunge la ricchissima esperienza, per secoli non sfruttata né utilizzata, che si genera scambiando informazioni, tessendo quella rete immateriale che in fondo è la struttura della cultura e del modo di vivere "civile" dell'intera umanità. Le piste associative sono le potenzialità (virtualità) che "attraversano" i materiali scritti, che li aprono oltre la loro dimensione fisica, la loro materialità.

I testi aperti non sono finiti e sono continuamente attualizzati e riutilizzati dagli utenti che guadagnano quindi un ruolo strategico. Sono i protagonisti di nuove forme di produzione intellettuale e culturale molto più ampie e molto più diffuse della cultura ufficiale, ristretta nel canone e nella tradizione del libro. Sono generatori di contenuti e contemporaneamente creatori di relazioni culturali e sociali fuori dalle regole istituzionali. È una previsione che anticipa quella che sarà la realizzazione vincente nel mondo delle reti: i social network e gli user generated content (UGC).

Esperienza e conoscenza sono i motori di questa nuova vision del rapporto tra tecnologie dell'informazione e della comunicazione e sapere (conoscenza più esperienza): “Appariranno enciclopedie di concezione radicalmente nuova, dotate di una trama di piste associative che le attraversano, pronte per essere inserite nel memex e lì arricchite di altre conoscenze" (Bush 1945; trad. it. Nelson Literary Machines, 1/51).

È un vero cambio di paradigma che diventerà molto più evidente negli anni successivi quando si porrà sempre più attenzione alle connessioni e alle relazioni tra le informazioni. Scout, scouting, explorer, trials sono le parole chiave di Bush:

Nascerà la nuova professione di battitore di piste [trials], persone che si ingegneranno a tracciare piste significative attraverso l'immane mole dell'esperienza umana. L'eredità del maestro ai suoi discepoli non saranno più solo i suoi contributi alla conoscenza comune, ma l'intera impalcatura di conoscenze sulla quale essi sono stati costruiti. (Bush 1945; trad. it. Nelson Literay Machines $1 / 52)$

I processi associativi rinviano ai meccanismi mentali "naturali," non a quella mente alfabetica (Havelock) che contraddistingue l'uomo occidentale a partire dall'invenzione della scrittura e soprattutto della stampa. La tecnologia della scrittura e ancor più quella della stampa hanno determinato il nostro modo di conoscere il mondo e di interagire con esso; progettare e poi costruire strumenti e quindi media che stabiliscano un altro rapporto col mondo, un ritorno ai flussi e alle associazioni che erano propri dell'oralità vuol dire andare oltre la gabbia gutemberghiana che produce il modello logico-sequenziale. Vuol dire andare oltre il testo come forma dominante nella civiltà moderna. Connessione diventa parola chiave per tutto il mondo del computing futuro e di quello dei network. 


\section{Augmenting intellect}

Douglas Engelbart scrive A conceptual Framework for the augmentation of Man's intellect nel 1963, svolgendo la tesi secondo cui il computer è uno strumento per potenziare l'intelligenza umana; il sistema proposto da Engelbart si chiamava H-LAM/T (Human using Language, Artifacts and Methodology, in which he is Trained). Engelbart sostiene, infatti, che utente e computer sono componenti che mutano dinamicamente, sempre in simbiosi, con l'effetto di potenziare l'intelligenza iniziale dell'utente. Nel 1968 Engelbart, durante la Fall joint computer conference diede una prima dimostrazione concreta del suo sistema, che aveva chiamato, molto significativamente, "Augment" e in quella occasione parlò di "lavoratori della conoscenza." Il mouse è un passo decisivo per la costruzione degli ipertesti, così si realizza un modo di "navigare” nell'ipertesto diverso dalla lettura lineare e sequenziale. Invece di leggere parola per parola o riga per riga oppure scorrere con l'occhio, ma sempre in sequenza, secondo un percorso lineare; nell'ipertesto, con l'uso del mouse, si può "saltare" da un documento all'altro, anche di notevole dimensione, senza vincoli prefissati e, sempre col mouse, è possibile fare apparire sullo schermo testi, immagini, suoni “annidati sotto” l'icona o il segnale predisposto. Secondo Engelbart questo procedimento, agevolato dalla tecnologia informatica, favorisce lo sviluppo delle capacità intellettive dell'uomo, specialmente nel campo dell'elaborazione "astratta”, trasformando il computer da strumento di solo calcolo in macchina per l'elaborazione di simboli. Per Engelbart, dunque, il computer è soprattutto un manipolatore di simboli; e su questa strada procede, realizzando nel 1968 l'NLS (On Line System), un sistema indirizzato a supportare la gestione complessa di più documenti in relazione tra loro.

\section{Xanadu: Dream contra Intellect}

Ted Nelson, autore del progetto XANADU, privilegia la creazione di "un ambiente letterario unificato su scala globale"; conia il termine "ipertesto", affermando: "sotto la guida di idee che non sono tecniche ma letterarie stiamo implementando un sistema per la memorizzazione e il reperimento di testi collegati tra loro e visibili in finestre. Il documento..."può avere finestre di qualsiasi altro documento" (Nelson, Literary Machines 3/2-7). L'obiettivo è quello di poter disporre on line di tutta la letteratura mondiale. Nelson, che a quel tempo lavorava alla Brown University, propose nel suo Computer lib/Dream machines (La biblioteca informatica ovvero le macchine per sognare) una serie di indicazioni precise, dedicate a quello che lui definì un ipertesto per visionari.

L'atteggiamento di Nelson verso la macchina può essere ben compreso da una sorta di autobiografia che appare in The future of information. Nelson ponendo al centro del suo interesse la scrittura come progettazione, come invenzione e elaborazione di idee, immagina il passaggio dalla funzione tipica della macchina da scrivere, cioè meccanizzare le funzioni di scrittura, a una, cognitiva e inventiva (creativa) da realizzare attraverso il computer e in particolare attraversale tecnologie ipertestuali. Nelson rafforza in modo esplicito questa posizione richiamando la propria formazione. Questa formazione favorisce un approccio non soltanto tecnico al computer. 
Alcuni hanno detto che io non capisco i computer perché credo che le cose dovrebbero essere molto differenti. Questo mi fa rabbia; non credo di sbagliare se dico che forse io capisco i computer meglio di loro.

Il mio pensiero è molto differente da quello delle altre persone del mondo dei computer, ed è per questo che progetto le cose a modo mio. [...] Queste idee non mi giungono da alcuna formazione scientifica o tecnologica, ma da un retroterra di scrittura teatrale e di realizzazione di film che risale alla mia infanzia e alla mia famiglia. Quando per la prima volta capii che cosa c'è dentro un computer, e che si può fargli fare assolutamente qualsiasi cosa, immediatamente pensai ai computer per la scrittura, per il teatro e per la realizzazione di film. Ciò accadeva nel 1960, molto prima che fosse stato inventato il termine «word processing» e molto prima che chiunque possedesse un computer personale. Ma per me era evidente che noi tutti avremmo letto e scritto su schermi, e che il problema era di progettare i nostri media del futuro per lo schermo del computer. (È impossibile per un lettore giovane immaginare come tutto ciò apparisse una pazzia a chiunque incontravo, per molti anni). Ma l'approccio parallelo fianco a fianco della mia progettazione di schermi interattivi iniziò allora. (Nelson, The Future of Information, 12; trad. mia)

Il carattere "visionario” di Nelson appare in piena luce; è evidente l’importanza che viene attribuita all'esperienza dei media e ai loro linguaggi e alle loro inter-relazioni; qui, quasi naturalmente, il computer è un medium che offre soluzioni insperate e opportunità eccezionali creando attraverso lo schermo un mondo e un ambiente che Nelson stesso descrive come "interattivo": l'incontro tra le tecnologie dell'informazione e i linguaggi dei media del Novecento è il carattere centrale di questa esperienza e delle proposte che caratterizzeranno l'opera di Nelson. Il computer è o può essere anche una macchina “espressiva” e non soltanto una macchina che elabora simboli.

Il riferimento che ritroveremo più volte alla letteratura (intesa da Nelson nel senso più ampio di produzione di documenti e di messaggi scritti) ${ }^{4}$ rimette al centro dell'attenzione alcune parole chiave: stile, espressività, apertura e pluralismo. Tutte categorie costitutive di un modello dinamico della creatività umana. Il computer diventa un compu-medium poiché si affianca, con-partecipa del processo creativo e inventivo; non è soltanto lo strumento che esegue con più efficacia dell'umano il compito che gli è stato assegnato: nel progetto Xanadu si progetta un insieme in cui il documento non è più un'unità isolata, autonoma, ma al contrario vive ed è fruibile proprio per le relazioni che incessantemente rivela e produce. Sono le connessioni, i links le relazioni che rendono vivo e operante questo prodotto universale delle attività culturali del genere umano. L'inter-relazione tra i documenti e la loro reciproca interdipendenza riflette un'azione degli utenti “comunitaria” e sociale.

Pur essendo considerato uno dei principali ispiratori dello sviluppo degli ambienti di rete fino al World Wide Web e pur riconoscendosi in alcuni suoi principi fondamentali, Nelson critica aspramente le soluzioni che progressivamente si impongono nella gestione degli ambienti Web: 
Il Progetto Xanadu, il progetto originale dell'ipertesto, è spesso scambiato per un tentativo di creare il World Wide Web. Esso ha sempre avuto obiettivi più ambiziosi, proponendo una forma di letteratura interamente nuova in cui i collegamenti non si rompono quando le versioni cambiano; dove i documenti possono essere confrontati da vicino, fianco a fianco, e fittamente annotati; dove è possibile vedere le origini di ogni citazione; e in cui c'è un valido sistema di copyright - un accordo letterario, legale ed economico - per citazioni senza ostacoli, non negoziate, in qualsiasi momento e in qualsiasi misura. Il web ha banalizzato il modello originale di Xanadu, semplificando ampiamente ma erroneamente questi problemi con la creazione di un mondo di fragili collegamenti monodirezionali sempre guasti, senza possibilità di riconoscere cambiamenti o copyright, e senza supporto per versioni multiple o riuso regolamentato. Prevalgono caratteri [fonts] e brillantini [glitz] anziché una struttura di connessione dei contenuti. (Nelson, Xanalogical Structures; trad. mia)

Creare documenti elettronici “profondi”, arricchirli continuamente, favorire lo scambio tra gli utenti che comunicano i loro documenti potendo sempre riconoscere l'originale, spinge Nelson a utilizzare l'immagine tradizionale della letteratura in modo nuovo, come infinito serbatoio continuamente rinnovato e continuamente rinnovantesi di messaggi. Una letteratura che non ha più connotazioni disciplinari, che non sta dentro il canone, e che non è più costituita soltanto da opere prodotte da autori riconosciuti dalla critica o dalla tradizione stessa.

Nella tradizione ci sono scrittori e lettori che comunicano attraverso i documenti e la letteratura. È una tradizione che privilegia il modello lineare e sequenziale e insieme quello gerarchico. Nell'idea ipertestuale di Nelson emerge tutto quel sapere e quella conoscenza che si genera dalle relazioni dei fruitori (lettori, co-autori, ecc.). In queste relazioni la funzione del testo o del documento può essere solo strumentale o occasionale. Le connessioni e le relazioni assumono un valore per sé. Questo è il nocciolo rivoluzionario delle comunità virtuali che così si generano; l'utopia è orientata alla costruzione di un sistema generale di comunicazione e di un insieme di forme espressive che possano svilupparsi liberamente, ma soprattutto interagendo. Nelson sostiene la libertà di comunicazione come base delle diverse forme di evoluzione della rete, ma soprattutto guarda allo sviluppo di un sistema universale come furono in età passate la scrittura alfabetica e poi il libro a stampa. La nuova tecnologia che può sostenere questa impresa è rappresentata sì dal computer, ma insieme da una nuova idea delle sue funzioni; la tecnologia ritorna al servizio dell'invenzione, della creatività, della produzione di significati:

Ad esempio, siamo alla ricerca di metodi che ci consentano di manipolare, sempre che riusciamo a scoprire come definirla, la "vera" struttura di un testo. [...] Ciò che noi forniremo è un servizio per immagazzinare e reperire porzioni arbitrarie di questa "vera” struttura [...] la struttura di cui stiamo parlando è la letteratura. (Nelson, Literary Machines, 2/4) 
In questo modo Nelson ritorna alle sue origini di scrittura drammaturgica, di ricerca di forme espressive sperimentali. Se rileggiamo alcune sue affermazioni possiamo notare accenti che rinviano a una stagione artistica caratterizzata dalle avanguardie del Novecento, a autori come Pirandello (la realtà non conclude), ai surrealisti e alle forme di sperimentazione linguistica e comunicativa (si pensi alla tecnica del collage), infine agli scrittori dello stream of conscousness, primo fra tutti James Joyce:

Non esiste l'Ultima Parola. Non può esistere la versione finale di qualcosa, l'ultimo pensiero. C’è sempre un nuovo punto di vista, una nuova idea, una reinterpretazione. E la letteratura, che noi proponiamo di trasporre su supporti elettronici, è un sistema per salvaguardare la continuità alla luce di questo fatto [...] Ricordatevi l'analogia fra il testo e l'acqua. L’acqua scorre liberamente, il ghiaccio no. I documenti vivi che scorrono liberamente attraverso la rete sono continuamente soggetti a nuovi utilizzi e all'aggancio di nuovi collegamenti, e questi nuovi collegamenti sono sempre disponibili per l'interazione. Ogni copia separata dal sistema è invece fredda e morta, manca di qualsiasi accesso ai nuovi collegamenti. (Nelson, Literary Machines, 2/57-58, 2/44)

Da qui anche la difficoltà per ora insormontabile per Nelson: costruire Xanadu vuol dire porsi il problema di nuove forme di comunicazione quindi di alfabeti, grammatiche, tecniche forme espressive che sono attualmente al di fuori del canone e dei modelli quotidiani in uso. Siamo ai limiti come il Finnegans Wake di Joyce o certa pittura informale.

Il luogo ideale per realizzare questo sistema totale e in movimento non è più la superficie piatta, unidirezionale che proviene dalla cultura della modernità e della stampa: la profondità e la complessità e le dinamiche dei significati e delle loro correlazioni vengono messe in scena sullo schermo. Lo schermo del computer è il luogo immateriale dove si possono rappresentare questi processi: lo schermo è la scena, è il luogo dell'azione, ambiente in cui vivono e si agitano i protagonisti.

Questa idea della complessità dello schermo, non più soltanto finestra sul mondo ma ambiente in cui i soggetti umani vivono e rappresentano anche i loro mondi interiori, sarà un tema forte di altri protagonisti di quella stagione straordinaria tra la fine degli anni Sessanta e l'inizio degli anni Ottanta del Novecento in USA, caratterizzata dai contributi di alcune donne come Brenda Laurel e il suo saggio Computer as Theatre e poi Sherry Turkle con Life on the Screen. Tutte tese a superare la meccanicità di un'idea positivista della tecnologia, a passare oltre a tutte le forme di determinismo per riprendere la strada del primato delle forme autentiche e espressive dell'umano nel confronto ormai ineludibile con la nuova macchina e con i nuovo ambienti di rete. 
1 "HyperCard è un software per creare ipertesti che comprende un linguaggio di programmazione introdotto sul mercato da Apple Computer nel 1987, assieme al System 6. Fu ritirato dalle vendite nel marzo del 2004. A differenza dei database tradizionali HyperCard è un sistema ipertestuale basato su concetti grafici, molto flessibile e molto semplice da modificare. In più, contiene il linguaggio di programmazione HyperTalk, semplice ma potente, per manipolare dati e interfaccia grafica. HyperCard fu creato da Bill Atkinson che lo diede ad Apple dietro la promessa di rilasciarlo al mercato in modo gratuito (promessa che, secondo Atkinson, non fu mantenuta quando venne messa in vendita la versione 2.0, largamente rimaneggiata)." La breve storia di HyperCard, disponibile su http://it.wikipedia.org/wiki/HyperCard, conferma la nostra tesi: il software fu un prodotto fortemente innovativo che apriva uno spazio - allora impensabile - all'utente. L'utente non programmatore professionale poteva usarlo piuttosto facilmente e si rivelò una nuova strada per i pc e la rete. Ma la contraddizione e quindi il conflitto emerge: aprire all'utente non è soltanto un atto tecnologico, è anche una scelta culturale, economica e sociale. Il prodotto è gratuito nelle intenzioni dell'inventore o comunque non ha margini di profitto. E quindi Apple, l'azienda madre della creatività, lo abbandona senza rimpianti!

${ }^{2}$ Bolter in Lo spazio dello scrivere afferma che la stampa ci ha incoraggiati a pensare al testo scritto come a una "creazione immutabile, un monumento al suo autore e alla sua epoca" (Bolter 13). Lo studioso ricorda come Hugo affermasse che la biblioteca era diventata "un sostituto della cattedrale" (Bolter 126) e in un altro passo scrive "ciò che è innaturale nella stampa diventa naturale nel mezzo elettronico, e presto non ci sarà nemmeno più bisogno di parlarne, perché lo si potrà mostrare” (Bolter 67). Ritorneremo su questo contrasto tra artificiosità e meccanicità della tecnologia gutemberghiana e "naturalità, fluidità" della tecnologia elettronica, prendendo lo spunto da As We May Think di Vannevar Bush.

3 L'articolo di Bush apparve sulla rivista Atlantic Monthly nel luglio del 1945. Due ricercatori, James M. Nyce e Paul Kahn, hanno dimostrato che Bush nel 1937 ne aveva scritto una versione assai più ampia. A questo proposito si veda, degli stessi autori, "Innovation, Pragmatism, and Technological Continuity: Vannevar Bush's Memex". Un'accurata ricostruzione del percorso di Vannevar Bush, corredata di interventi tra i maggiori "prosecutori" della sua linea come Nelson o Engelbart si trova in Nyce James M., e Kahn, eds. From Memex to Hypertext.

${ }^{4}$ Più propriamente Nelson con letteratura intende l'insieme dei testi (o documenti) scritti e non soltanto quelli provvisti di "letterarietà" (e quindi riconosciuti nella tradizione letteraria); cfr. Nelson, Literary Machines, 2/8. 


\section{Works Cited}

Bolter, Jay David. Lo spazio dello scrivere. Computer, ipertesto e la ri-mediazione della stampa. Milano: Vita e Pensiero, 2002. Print.

Bush, Vannevar. "As we may think.” Atlantic Monthly, July 1945: 101-108. Print.

Delany, Paul and Landow George, Eds. Hypermedia and Literary Studies. Cambridge (Mass.): MIT Press, 1991. Print.

De Sanctis, Francesco. Storia della letteratura italiana. Napoli: Morano 1870. Print.

Derrida, Jacques. L'écriture et la différence. Paris: Seuil, 1967; La scrittura e la differenza. Trans. Giovanni Pozzi. Torino: Einaudi, 1971. Print.

Engelbart, Douglas C. "A Conceptual Framework for the Augmentation of Man's Intellect”. Vistas in Information Handling. Vol. 1. Eds. Paul Howerton and David Weeks. Washington, D. C.: Spartan Books, 1963. 1-29.

Goody, Jack. La logica della scrittura e l'organizzazione della società. Torino: Einaudi, 1988. Print.

---. Il suono e i segni: l'interfaccia tra scrittura e oralità. Milano: Il Saggiatore, 1987. Print.

Havelock, Eric A. La Musa impara a scrivere: riflessioni sull'oralità e l'alfabetismo dall'antichità al giorno d'oggi. Roma: Laterza, 1997. Print.

---. Oralità e scrittura. Le tecnologie della parola. Bologna: Il Mulino, 1986. Print.

Landow, George P. L'ipertesto. Tecnologie digitali e critica letteraria. A cura di Paolo Ferri. Milano: Bruno Mondadori, 2000. Print.

Laurel, Brenda. Computer as Theatre. Reading (Mass.): Addison-Wesley, 1991. Print.

Nelson, Theodor H. Computer Lib; Dream Machines. Redmond, Wash: Tempus Books of Microsoft Press, 1987. Print.

---. Literary Machines 90.1. Padova, Muzzio, 1992. Print.

---. The Future of Information. Tokyo: ASCII, 1997. Print.

---. "Xanalogical structure, needed now more than ever: parallel documents, deep links to content, deep versioning, and deep re-use." ACM Computing Surveys 31(4),December,1999.Web.

<http://www.cs.brown.edu/memex/ACM HypertextTestbed/papers/60.html>

Nyce James M., e Kahn, Paul. "Innovation, Pragmatism, and Technological Continuity: Vannevar Bush's Memex." Journal of the American Association for Information Science, 40, (1989): 214-22. Print.

Nyce James M., e Kahn, Paul. Eds. From Memex to Hypertext. San Diego: Academic Press Inc., 1991; trad. it, Franco Padova: Muzzio, 1992. 
Marx Karl. Manoscritti economico-filosofici del 1844. Torino: Einaudi, 1949. Print.

Ong, Walter. J. Oralità e scrittura. Le tecnologie della parola. Bologna: Il Mulino, 1986. Print

Turkle, Sherry. La vita sullo schermo. Nuove identità e relazioni sociali nell'epoca di Internet. Con un saggio introduttivo di Mario Ricciardi. Milano: Apogeo, 2005. Print.

Wikipedia. "HyperCard." Wikipedia, L'enciclopedia libera. Web.

<http://it.wikipedia.org/w/index.php?title=HyperCard\&oldid=51572372>. 